\title{
A Haptic-Enhanced System for Molecular Sensing
}

\author{
Sara Comai and Davide Mazza \\ Politecnico di Milano \\ Department of Electronics and Information (DEI) \\ Piazza L. da Vinci 32 \\ I-20133 Milan \\ \{comai,mazza\}@elet.polimi.it
}

\begin{abstract}
The science of haptics has received an enormous attention in the last decade. One of the major application trends of haptics technology is data visualization and training. In this paper, we present a haptically-enhanced system for manipulation and tactile exploration of molecules. The geometrical models of molecules is extracted either from theoretical or empirical data using file formats widely adopted in chemical and biological fields. The addition of information computed with computational chemistry tools, allows users to feel the interaction forces between an explored molecule and a charge associated to the haptic device, and to visualize a huge amount of numerical data in a more comprehensible way. The developed tool can be used either for teaching or research purposes due to its high reliance on both theoretical and experimental data.
\end{abstract}

\section{Introduction}

Molecular analysis has acquired a great importance in recent years. The study of interactions among molecules and the ways in which they can be aggregated are at the basis of drug design. A deep understanding of the inter-molecular forces that govern this kind of processes is fundamental. Interactions among molecules are usually described by huge sequences of data, which describe the attraction/repulsion forces and the positions of interesting sites for binding over molecular surfaces in a very detailed way, but awkward to interpret $a s$-is even by scientists and chemists of the field. Haptic interaction could greatly help here, because the sense of involved forces could be used to better understand the intensity of interactions that humans are not able to feel in everyday life, due to their existence at nanoscale or atomic levels. In this way, scientists could reach a better awareness on the location and the nature of these interactions, that could be exploited in further experimental studies.

At this aim we developed a virtual environment, where the molecule to be analyzed is shown to the user and a probing charge is associated with a haptic device. The interaction between the molecule and the electric charge is felt via the haptic device and allows one to explore the electrostatic surface of the molecule. This paper is organized as follows: Section 2 provides an overview of the system and shows how information and results are rendered; Section 3 reports feedbacks obtained by users; Section 4 presents related works, and, finally, Section 5 draws our conclusions and future plans. 


\section{Description of the Haptic-Enhanced System}

The developed system takes in input the geometrical representation of the molecule in terms of theoretical model (.PDB file format) or experimental data (.CIF file format, obtained from crystallography). For a reference on PDB and CIF file format see [5, 3]. Such data are then elaborated by a computational chemistry tool [4], which provides in output the electric field data needed to model the force interaction. Figure 1 sketches the architecture of the developed system.

Both input and output data are huge in size and it is therefore difficult to catch atoms position and electronic fields' interactions directly from such data. Figure 2 shows some examples of fragments of input and output data. Just to give an idea of the amount of information to deal with, the original files shown in Figure 2, referred to the ammonia molecule, are composed of 1508 and 22933 lines of numbers!

The developed system provides a virtual environment showing a $3 \mathrm{D}$ representation of the molecule. Different visualizations are possible, like, for example, space-filling and ball-and-stick. Beside providing the graphical representation, the system allows to feel the electronic fields' interactions by means of a haptic device (in our case, the Sensable PHANTOM® Omni [6]): the graphical environment shows the current position of the haptic proxy, to indicate which part of the space the user is interacting with. Auxiliary key information are also shown in an intuitive way, such as a plot of the electrostatic field along the direction connecting the proxy position and the center

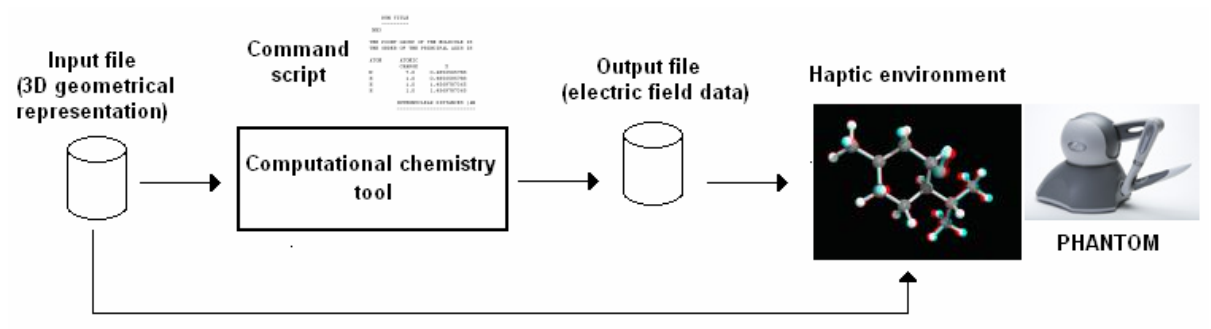

Fig. 1. The architecture of the designed haptic system
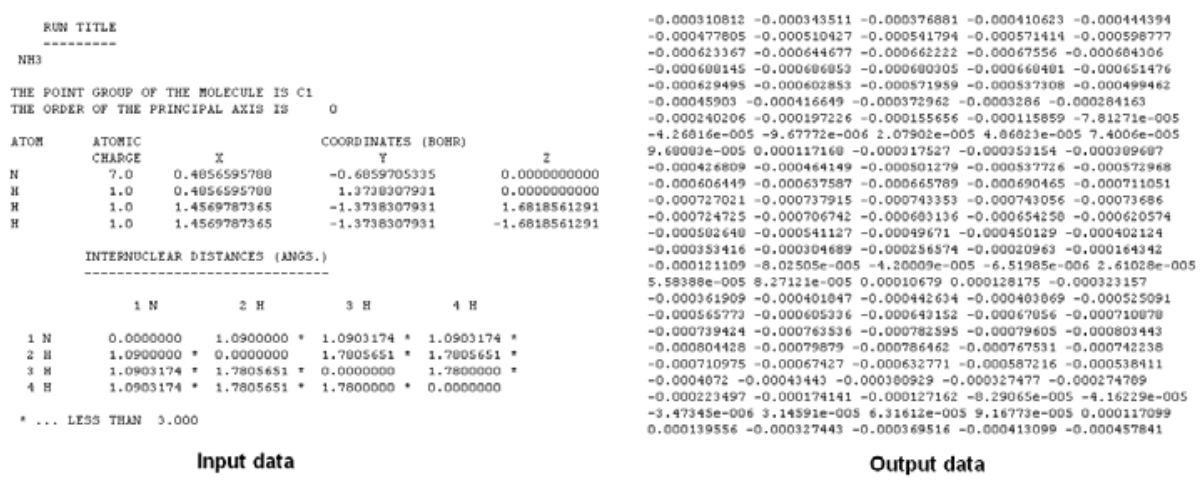

Fig. 2. Abstracts from sample input and output data ${ }^{-}$les in case of ammonia (NH3) molecule 


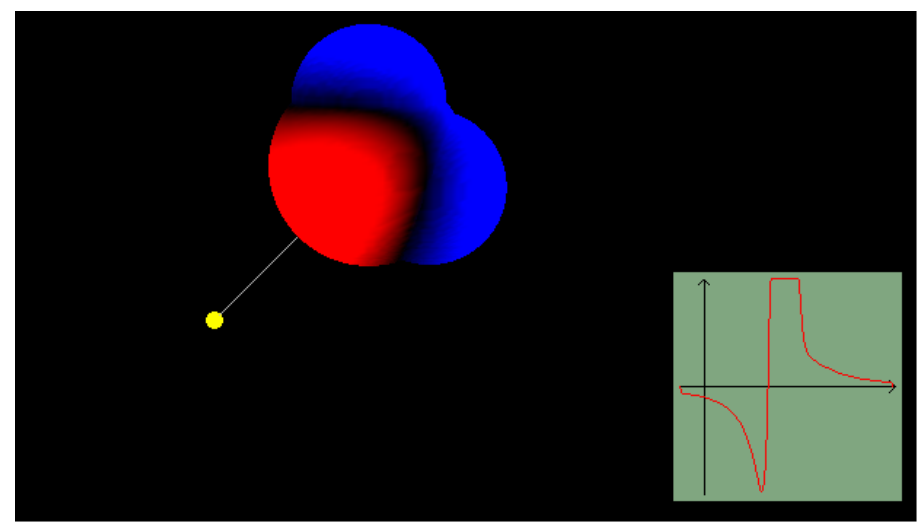

Fig. 3. A screenshot of the tool in case of water molecule. The proxy position (yellow circle) and the plot of the electrostatic field are visible. The plot is computed using the value of the field along the thin white line connecting the proxy with the center of the molecule.

of the molecule, and the force field on the molecule surface (represented by means of different colors). Figure 3 shows a snapshot of the tool in action. The 3D molecule representation and the proxy pointer are clearly visible. The plot of the electrostatic field is shown in the bottom-right corner.

The electrostatic field is represented by a quantized grid surrounding the molecule till a certain distance. The force felt by the user is the one associated with the voxel of the grid within which proxy position falls in. The forces are meant as opposed to probe movement done by the user and consists of attraction/repulsion forces according to the situation. Their intensity are naturally very weak (nanoscale). A human operator cannot feel them as is. After some usage tests, an amplification has been determined to scale the force appropriately without losing the proportions among real forces.

\section{Users' Feedbacks}

The tool has been tested by different kinds of users on different molecules (e.g., water, ammonia, methane). The students of a chemistry course tried the experience of the interaction as a didactic tool; chemical technicians appreciated the improved awareness of the phenomena they are used to work with, which are otherwise not so intuitive; researchers tried to test how the tool is able to render well-known interactions. We have tested the tool on molecules with known electrostatic behaviour and interesting binding sites, and checked with chemical experts the obtained behavior to confirm that the simulation is correct. In general, the most important benefit is represented by the possibility of combining the visualization of data with the rendering of the feeling of nanoscale/atomic interactions that can improve the understanding of real phenomena.

\section{Related Works}

[7] provides a survey on haptic rendering techniques: most of the haptic algorithms and applications focus on the rendering of surface tactile feedback, rather than forces 
distributed in the whole space as this does. In the field of chemical visualization and haptics, our tool is a border-line one and stands along the boundary of old computational chemistry (which privileges awkward numerical outputs, disregarding userfriendliness) and the brand-new bioinformatics (which introduces better and friendly UIs to show results). Most of the developed tools, whenever presenting haptic interaction, exhibit it as a side feature, usually because it has been added later. In our tool it is the core interaction. Examples of similar tools include VMD, PyMol, and others (see [2] for a survey), which offer a wide range of representations, but interpretation of data is not so straightforward for non-technician of the field. [1] presents a similar tool, but it is strongly designed on theoretical models and, compared to our systems, it does not allow to use or explore empirical data directly obtained in laboratories.

\section{Conclusions and Future Work}

This work is meant as the first step towards the development of a framework for the simulation of the interaction between complex chemical compounds, such as a protein and another -whatever complex- molecule and is carried on in collaboration with the Chemical Department of our University. In particular the focus will be on molecules exhibiting halogen bonding, a kind of ligand known in literature since 60s but arisen again in recent years due to its importance in drug design. Further improvements include the implementation of interactions of pairs of halogenated compounds, either from a statical or dynamical point of view, to better understand the behavior of such bonding and to allow the usage of the tool in scientific/research frameworks.

Acknowledgements. We would like to thank Pierangelo Metrangolo (chemistry), Antonino Famulari (chemistry), Marzio Ghezzi (labs and device) and Marco Faverio (development) for their precious contributions.

\section{References}

1. Chemical force feedback, http://cff.itn.liu.se/public/

2. Chemistry visualization software survey, http://personal.cscs.ch/mvalle/ChemViz/tools.html

3. The cif file form, http: / / www.wrcad.com/manual/xicmanual/node493.html

4. The general atomic and molecular electronic structure system (gamess), http: / / www.msg.ameslab.gov/GAMESS /

5. Pdb file format specifications, http://deposit.rcsb.org/adit/docs/pdbatomformat.html

6. Sensable Phantom ${ }^{\circledR}$ Omni, http: / /www. sensable.com/haptic-phantom-omni.htm

7. Laycock, S., Day, A.: A survey of haptic rendering techniques. Computer Graphics forum 26(1), 50-65 (2007) 